\title{
Predicting Fetal Distress and Admission to Neonatal Intensive Care Unit in Patients With Fetal Growth Restriction by Nomogram: A Retrospective Cohort Study in China
}

\section{Xiaobin Chen}

Women's Hospital School Of Medicine Zhejiang University

Yuan Chen

Women's Hospital School Of Medicine Zhejiang University

Ying Jiang

Women's Hospital School Of Medicine Zhejiang University

Tian Dong

Women's Hospital School Of Medicine Zhejiang University

Juan Li

Women's Hospital School Of Medicine Zhejiang University

Mengmeng Yang

Women's Hospital School Of Medicine Zhejiang University

\section{Baihui Zhao}

Women's Hospital School Of Medicine Zhejiang University

Qiong Luo ( $\square$ luoq@zju.edu.cn )

Women's Hospital School Of Medicine Zhejiang University

\section{Research Article}

Keywords: Fetal distress, Neonatal intensive care unit, Fetal growth restriction, prediction model, Nomogram

Posted Date: December 4th, 2020

DOl: https://doi.org/10.21203/rs.3.rs-115166/v1

License: (9) (1) This work is licensed under a Creative Commons Attribution 4.0 International License. Read Full License 


\title{
Predicting fetal distress and admission to neonatal intensive care unit in patients with fetal growth restriction by nomogram: A retrospective cohort study in China
}

\author{
Xiaobin Chen, Yuan Chen, Ying Jiang, Tian Dong, Juan Li, Mengmeng \\ Yang, Baihui Zhao, Qiong Luo
}

\begin{abstract}
Background: The purpose of this research was to establish prediction model of fetal distress risk and admission to neonatal intensive care unit(NICU) risk in a Chinese population of patients with fetal growth restriction(FGR), to provide a reliable basis for clinical management.
\end{abstract}

Methods: A retrospective analysis of 930 patients were delivered with FGR in the obstetrics and gynecology hospital affiliated from January 1, 2014 to December 30, 2019 at Women's Hospital, School of Medicine, Zhejiang University, in Hangzhou, and screened out the independent predictors affecting fetal distress and admission to NICU using lasso regression. Multivariable logistic regression analysis was used to establish the nomogram prediction model of fetal distress risk and admission to NICU risk. Discrimination of the predicting model was assessed by the C-index and receiver operating characteristic (ROC) curve, using the bootstrapping validation and the ROC curve to evaluate the Internal validation. Calibration and clinical usability of the predicting model were respectively adopted by calibration curves and decision curve analysis. Internal validation was assessed using the bootstrapping validation.

Results: We found that six identified factors associated with fetal distress of patients with FGR: age, gestational week, delivery method, placenta previa, abnormal cord blood flow, and using low molecular weight heparin(LMWH). The application of LMWH during pregnancy could reduce the incidence of fetal distress. Four independent predictors were selected for admission to NICU of patients with FGR: gestational week, delivery method, hypertensive disorders of pregnancy, and intrahepatic cholestasis of pregnancy. The delivery method of cesarean section increased the above risks. Two nomograms were developed and verified accordingly. The two models had good discrimination and good calibration respectively. The decision curve analysis performed that the clinical usability and benefits of the nomograms were the range of 3\%-75\% and $17 \%-95 \%$.

Conclusions: Two nomograms were the first to predict fetal distress and admission to NICU of patients with FGR. Establishing effective predictive models based on independent predictors could help early diagnosis and evaluation of fetal distress and admission to NICU in patients with FGR.

Keywords: Fetal distress, Neonatal intensive care unit, Fetal growth restriction, prediction model, 
Nomogram

\section{Background}

Fetal growth restriction (FGR) is one of the common complications of obstetrics and the second leading cause of perinatal death. It has specific clinical features, short-term and long-term risks[1-3]. Current research showed that its occurrence was affected by many factors, including maternal factors [4], placental and umbilical cord factors[4, 5], fetal factors[6], genetic factors[7-9], etc.

Fetal factors included multiple pregnancies, fetal chromosomal abnormalities, fetal malformations and other factors, and clinical intervention was of little significance. Owing to lack of effective treatment, the outcome of fetus was the early termination of pregnancy. However, focusing on maternal diseases, placenta and umbilical cord factors, there are currently a large number of studies to improve the prognosis of the fetus for FGR patients at risk of adverse outcome[5, 10,11], but there were lack of effective predictive models to predict the occurrence of fetal distress and admission to neonatal intensive care unit (NICU) for FGR patients. Therefore, our research constructed two new predictive models. A visual and simple nomogram evaluated its risk and provided more reliable evidence for the next active treatment.

\section{Methods}

\section{Patients' characteristics}

Retrospective analysis of 930 patients were delivered with FGR in the obstetrics and gynecology hospital affiliated from January 1, 2014 to December 30, 2019 at Women's Hospital, School of Medicine, Zhejiang University, in Hangzhou. We selected maternal features as follows: age, pregnancy times, birth times, body mass index (BMI) before delivery, weight gain during pregnancy, gestational week, delivery method, hypertensive disorders of pregnancy(HDP), intrahepatic cholestasis of pregnancy(ICP), gestational diabetes mellitus(GDM), hypothyroidism, Placenta previa, thrombosis, anticardiolipin syndrome, abnormal cord blood flow(absent or upside down), oligohydramnios, recurrent spontaneous abortion (RSA), using prednisone, using aspirin, using low molecular weight heparin. The following information of the offspring was obtained: sex, fetal distress, admission to NICU. We selected information about fetal distress diagnosed by obstetricians and admission to NICU from the medical records of the hospital, as the outcome of the study. The most widely adopted diagnostic criteria of fetal FGR is an estimated fetal weight (EFW) below the 10th percentile for the gestational age[2]. Exclusion criteria included multiple pregnancies, cases of incomplete information. Among them, there were 150 cases of fetal distress and 780 cases without fetal distress. There were 478 cases of neonates entering into the NICU and 452 cases as a control. Selecting $70 \%$ of the total cases randomly was for internal validation (Figure 1A).

\section{Ethics approval and consent to participate}


This study has been approved by the Ethics Committee of our hospital (Ethical batch number:IRB-20200283-R). All methods of our study were performed in accordance with the our country's guidelines and regulations, and patients informed consent for participation by signing in this study. They all volunteered to participate in this research, and the information obtained was used for this research only.

\section{Statistical analysis}

Statistical analyses of all data, which were represented by counting data, performed using $\mathrm{R}$ software (Version 3.6.0; https://www.R-project.org). The least absolute shrinkage and selection operator (LASSO) method[12] was used to screen out the clinical characteristics that it was best to predict the risk factors of fetal distress and entering into NICU, and then multivariate logistic regression analysis was used to build two predicting models of the risk factors of fetal distress and entering into NICU, $\mathrm{P}<0.05$ was considered statistically significant. Two predicting models of nomogram were formulated based on the results of logistic regression analysis and by using $R$ software. Discrimination of the two predicting models of nomogram were assessed by the concordance index (C-index) and receiver operating characteristic (ROC) curve. Bootstrapping validation with 1,000 resample were used for calculating a relatively corrected C-index. Selecting $70 \%$ of the total sample size randomly was as internal validation. Internal validation was assessed using the bootstrapping validation. Calibration and clinical usability of the two predicting models were respectively adopted by calibration curves and decision curve analysis. Decision curve analysis is a novel method that is better than the traditional decision analytic techniques to evaluate prediction models [13]. Using the ROC curve and calibration to execute the Internal validation.

\section{Results}

\section{Lasso regression analysis results}

We screened out the variables using the offspring outcome of fetal distress by lasso regression analysis. Six optimal variates were selected from the twenty-one variables as follows: age, gestational week, delivery method, placenta previa, abnormal cord blood flow (absent or upside down), LMWH (Figure $1 \mathrm{~B}$ and $\mathrm{C}$ ). Table1 lists the patients' characteristics of the Chinese population among the six independent predictors. According to the outcome of admission to $\mathrm{NICU}$, nine optimal variates were screened out among the twenty-one variables using lasso regression analysis, for instance pregnancy times, gestational week, delivery method, HDP, ICP, oligohydramnios, RSA, using prednisone, newborn sex (Figure 1D and E). Table2 lists the information of nine variables.

\section{Prediction model Construction}

Selecting six optimal variates about fetal distress risk were analyzed by multivariable logistic regression analysis. The results were given in Table 3, the prediction model that merged the above six independent predictors was presented using the nomogram (Figure 2A). Selecting nine 
optimal variates about the risk of admission to NICU were performed by multivariable logistic regression analysis. The results listed in Table4. The prediction model that amalgamated the above four independent predictors was revealed as the nomogram (Figure 2B).

\section{Predictive Accuracy of the Nomogram}

The C-index of the nomogram predicted by the risk of fetal distress was $0.733(95 \% \mathrm{Cl}: 0.690-$ 0.776)for total cases, and was acknowledged to be 0.718 through bootstrapping validation. Simultaneously, The C-index of the prediction nomogram of admission to NICU risk was 0.794 (95\% Cl:0.766- 0.822) for total cases, and was confirmed to be 0.792 through bootstrapping validation.

Furthermore, our models demonstrated good discriminative ability in both the total (AUC of prediction model one: 0.733 , Figure3A. AUC of prediction model two: 0.794, Figure 3B) and internal Validation (AUC of prediction model one: 0.727 , Figure3C. AUC of prediction model two: 0.801 , Figure $3 \mathrm{D}$ ) cases, which performed a good prediction capability in the risk of fetal distress and risk of admission to NICU nomogram.

\section{Calibration of the Nomogram}

The calibration curves of the prediction nomogram using to predict the fetal distress risk in FGR patients demonstrated a good agreement in the total (Figure 4A) and the internal Validation (Figure 4B) cases. At the same time, the calibration curves of the prediction of admission to NICU risk nomogram in FGR patients proved a good agreement in the total (Figure $4 \mathrm{C}$ ) and the internal Validation (Figure 4D) cases.

\section{Decision Curve Analysis of the Nomogram}

The decision curve analysis (DCA) was used to perform the clinical usability and benefits of the nomogram. As shown in Figure 5A, the DCA showed that if the threshold probability of fetal distress in a patient was the range of $3 \%-75 \%$, separately, using this fetal distress risk nomogram to predict the risk of fetal distress adds more benefit than the scheme. The DCA presented that if the threshold probability of admission to NICU was the range of $19 \%-97 \%$ in Figure $5 \mathrm{~B}$, respectively, using this the admission to NICU risk nomogram could predict the risk of admission to NICU adds more benefit than the scheme. Net benefit in this range was compared to several overlaps on basis of the fetal distress risk nomogram and admission to NICU risk nomogram.

\section{Discussion}

With the development of medical research in recent years, nomogram is a new type of multi-factor statistical method, which has better advantages than the total statistical method. It is widely applied in the medical fields and provides a visual basis for clinical work. Our study was the first to use nomogram to explore the risk of fetal distress and admission to NICU in patients with FGR in china. Using a novel prediction tool that was the lasso regression screened out more influential and available variables from the research factors in patients with FGR.

At present, FGR is the most concerned disease that affects fetal outcome in the field of fetal medicine [14]. In the early stage, Barker firstly proposed a hypothesis which was "fetal origins of 
adult hypothesis"[15, 16] . With the continuous research and development of the disease, it gradually transformed into "developmental origins of health and disease"[17, 18].An unhealthy maternal intrauterine environment not only affected the growth and development of the fetus, but also caused adverse consequences for the fetus[19]. Therefore, owing to its effective prenatal monitoring, timing and method of pregnancy termination are particularly important. However, there are still great controversies about FGR management due to the lack of effective and gold standard.

Our study started from the maternal disease to explore the outcome of the fetus, which has important clinical value. In the prediction model one, six independent variates were presented as predictors of fetal distress, and nine optimal predictors for staying into the NICU. With the increase of pregnant women's age, the decrease of gestational week, abnormal cord blood flow during pregnancy, the risk of fetal distress has increased in the fetus of FGR patients. Among them, the risk of fetal distress in FGR patients at the age of 40 years old was significantly higher than the others at the age of 35 years old ( $O R=4.058,95 \% \mathrm{Cl} 1.872-8.622, P<0.001)$. And abnormal cord blood flow also increased its risk obviously (OR $=7.563,95 \% \mathrm{Cl} 3.653-16.146, P<$ 0.001). FGR patients with placenta previa could reduce the occurrence of fetal distress (OR $=0.330,95 \% \mathrm{Cl} 0.094-0.878, P=0.046$ ). At present, there is no clear and sufficient evidence to prove that $\mathrm{LMWH}$ plays a role in the treatment of fetal growth restriction, so further research is still under way. Our study found that the application of LMWH during pregnancy could reduce the incidence of fetal distress ( $O R=0.554,95 \% \mathrm{Cl} 0.331-0.895, P=0.020$ ), but not affecting the risk of fetal entered into the NICU by lasso regression analysis. Therefore, we recommend that LMWH could reduce the incidence of fetal distress, which is consistent with the latest research results[3, 20]. For the use of clinical aspirin, a meta-analysis of research randomized controlled trials had shown that aspirin decreased the risk of fetal growth restriction[21]. We used the lasso regression to screen out variates, indicating that aspirin did not decrease the risk of fetal distress for fetal growth restriction. Our research tentatively studied that the gestational week decreases would increase the incidence of admission to NICU after birth $(P<0.001)$, which was similar to the research [22]. The fetus of FGR patients with HDP, ICP was easier to entry into the NICU $(P=0.032, P=0.011)$. The use of prednisone during pregnancy did not reduce the incidence of admission to $\operatorname{NICU}(P=0.120)$. For the more, it is interesting that children to be born for FGR patients whose sex is a boy was not significantly related to the incidence of staying in the $\mathrm{NICU}(P=0.244)$.

Regarding to the delivery method of FGR patients, it is not an absolute indication of cesarean section. When the cord blood flow was abnormal, it was recommended to terminate the pregnancy by cesarean section [23]. However, there was still a lot of controversy about the timing of delivery and the method of delivery in various countries [23-26]. The innovative findings of this study were that vaginal delivery, compared with cesarean delivery, could reduce the incidence of fetal distress and admission to NICU for FGR patients $(P<0.001, P<0.001))$. A foreign study showed that most patients with FGR achieved vaginal delivery, the terrible fetal outcome did not increase[27]. Even one research advocated vaginal delivery[28]. Therefore, we recommend that FGR patients chose vaginal delivery without serious emergency complications, and we monitored the labor process during delivery.

The independent predictors of two nomograms were developed based on prediction model one and two. We could add up the single points corresponding to the independent predictors of 
patients with FGR to get the total points. Finally, we got the probability of risk of fetal distress or admission to NICU. It was easier and more intuitive for clinicians to understand its risks. For example, the nomogram of prediction model two, a FGR patients of 34 gestational weeks (about 83 point), using the cesarean section to terminate pregnancy (about 40 point), without HDP ( 0 point) and ICP (0 point). The total points are 120 points, and the corresponding risk of admission to NICU is about $78 \%$. At the same time, when we verified two models, we found that these had good discrimination and calibration power. The internal verification results are consistent with the previous ones. When verified two models, we found that these had good discrimination and calibration power. The decision curve analysis suggested two models had better clinical application value. The internal verification results are consistent with the previous ones.

The current shortcoming of this study is that the timing of LMWH treatment and the timing of drug withdrawal was not studied, so further research is needed. In summary, the establishment of an effective predictive model is the key to prenatal management of the fetal outcome of FGR patients and provides a reliable basis for clinicians. The further treatment can reduce the occurrence of adverse maternal and infant outcomes.

\section{Conclusion}

Our research showed that six identified factors associated with fetal distress and four independent predictors were selected for admission to NICU of patients with FGR. The application of LMWH during pregnancy could reduce the incidence of fetal distress. The delivery method of cesarean section increased the above risks. Two nomograms were the first to developed and verified which had good discrimination and good calibration respectively. They are valuable for clinical prediction and practicality. Establishing effective predictive models based on independent predictors could help early diagnosis and evaluation of fetal distress and admission to NICU in patients with FGR.

\section{Abbreviations}

FGR:Fetal growth restriction; LMWH:low molecular weight heparin; HDP:hypertensive disorders of pregnancy, ICP:intrahepatic cholestasis of pregnancy; GDM:gestational diabetes mellitus, hypothyroidism; RSA:recurrent spontaneous abortion; NICU:neonatal intensive care unit; DCA:decision curve analysis; EFW:estimated fetal weight; LASSO:least absolute shrinkage and selection operator; C-index:concordance index; ROC:receiver operating characteristic.

\section{Acknowledgments}

We express our gratitude to the patients who participated in this study.

\section{Authors' contributions}

Xiaobin Chen and Yuan Chen contributed to the data analysis, chart making, writing- reviewing and editing of the study. Data management and curation were by Ying Jiang. Data collection was by Tian Dong. Chart making was by Juan Li. Software selection was by Mengmeng Yang. Baihui Zhao and Qiong Luo contributed to the concept, design, data collection, chart making, writing- 
reviewing and editing in the study and take responsibility for the accuracy of the data analysis.

\section{Funding}

This paper was supported by the National Key R\&D Program of China (2018YFC1002702), the Natural Science Foundation of Zhejiang Province of China (Grant No. LY2OH040009), the Key R\&D Program of Zhejiang Province of China (No.2018C03010) and Zhejiang Medical and Health Technology Project (No.2018244300).

\section{Availability of data and materials}

The data information can be obtained from the corresponding authors by E-mail( luoq@zju.edu.cn, zhaobh@zju.edu.cn)

\section{Ethics approval and consent to participate}

This paper was approved by medical Ethics Committee of Women's Hospital, Zhejiang University School of Medicine(Ethical batch number:IRB-20200283-R). All methods of our study were performed in accordance with the our country's guidelines and regulations, and patients informed consent for participation by signing in this study. They all volunteered to participate in this research, and the information obtained was used for this research only.

\section{Consent for publication}

Not applicable.

\section{Competing interests}

The authors have no competing interests to declare.

\section{Author details}

First authors are Xiaobin Chen and Yuan Chen. Corresponding authors are Qiong Luo and Baihui Zhao. Department of Obstetrics, Women's Hospital, School of Medicine, Zhejiang University, No.1, Xueshi Road, Shangcheng District, Hangzhou, China

\section{References}

1. Frøen JF, Gardosi JO, Thurmann A, Francis A, Stray-Pedersen B: Restricted fetal growth in sudden intrauterine unexplained death. Acta obstetricia et gynecologica Scandinavica 2004, 83(9):801-807.

2. ACOG: ACOG Practice Bulletin No. 204: Fetal Growth Restriction. Obstetrics and gynecology 2019, 133(2):e97-e109.

3. Martins JG, Biggio JR, Abuhamad A: Society for Maternal-Fetal Medicine (SMFM) Consult Series \#52: Diagnosis and Management of Fetal Growth Restriction. American journal of obstetrics and gynecology 2020, 223(4):B2-B17. 
4. Sharma D, Shastri S, Sharma P: Intrauterine Growth Restriction: Antenatal and Postnatal Aspects. Clinical medicine insights Pediatrics 2016, 10:67-83.

5. Nassr AA, Abdelmagied AM, Shazly SA: Fetal cerebro-placental ratio and adverse perinatal outcome: systematic review and meta-analysis of the association and diagnostic performance. Journal of perinatal medicine 2016, 44(2):249-256.

6. Denbow ML, Cox P, Taylor M, Hammal DM, Fisk NM: Placental angioarchitecture in monochorionic twin pregnancies: relationship to fetal growth, fetofetal transfusion syndrome, and pregnancy outcome. American journal of obstetrics and gynecology 2000, 182(2):417-426.

7. Chelbi ST, Wilson ML, Veillard AC, Ingles SA, Zhang J, Mondon F, Gascoin-Lachambre G, Doridot L, Mignot TM, Rebourcet R et al: Genetic and epigenetic mechanisms collaborate to control SERPINA3 expression and its association with placental diseases. Human molecular genetics 2012, 21(9):1968-1978.

8. Gascoin-Lachambre G, Buffat C, Rebourcet R, Chelbi ST, Rigourd V, Mondon F, Mignot TM, Legras $E$, Simeoni $U$, Vaiman $D$ et al: Cullins in human intra-uterine growth restriction: expressional and epigenetic alterations. Placenta 2010, 31(2):151-157.

9. Shen K, Moroco JA: The Src family kinase Fgr is a transforming oncoprotein that functions independently of SH3-SH2 domain regulation. 2018, 11(553): eaat5916.

10. Verfaille $V$, de Jonge $A$, Mokkink $L$, Westerneng $M$, van der Horst $H$, Jellema $P$, Franx $A$ : Multidisciplinary consensus on screening for, diagnosis and management of fetal growth restriction in the Netherlands. BMC pregnancy and childbirth 2017, 17(1):353.

11. Tanaka H, Furuhashi FH, Toriyabe K, Matsumoto T, Magawa S, Nii M, Watanabe J, Tanaka K, Umekawa $\mathrm{T}$, Kamimoto $\mathrm{Y}$ et al: Management of fetal growth restriction using the contraction stress test: a case-control study. The journal of maternal-fetal \& neonatal medicine : the official journal of the European Association of Perinatal Medicine, the Federation of Asia and Oceania Perinatal Societies, the International Society of Perinatal Obstet 2019, 32(19):3221-3225.

12. Kidd AC, McGettrick $M$, Tsim S, Halligan DL, Bylesjo $M$, Blyth KG: Survival prediction in mesothelioma using a scalable Lasso regression model: instructions for use and initial performance using clinical predictors. BMJ open respiratory research 2018, 5(1):e000240.

13. Vickers AJ, Cronin AM, Elkin EB, Gonen M: Extensions to decision curve analysis, a novel method for evaluating diagnostic tests, prediction models and molecular markers. $B M C$ medical informatics and decision making 2008, 8:53.

14. McCowan LM, Figueras F, Anderson NH: Evidence-based national guidelines for the management of suspected fetal growth restriction: comparison, consensus, and controversy. American journal of obstetrics and gynecology 2018, 218(2s):S855-s868.

15. Barker DJ, Osmond C: Infant mortality, childhood nutrition, and ischaemic heart disease in England and Wales. Lancet (London, England) 1986, 1(8489):1077-1081.

16. Walker AR, Walker BF: Fetal nutrition and cardiovascular disease in adult life. Lancet (London, England) 1993, 341(8857):1421.

17. Hanson MA, Gluckman PD: Developmental origins of health and disease: new insights. Basic \& clinical pharmacology \& toxicology 2008, 102(2):90-93.

18. Haas SA, Oi K: The developmental origins of health and disease in international perspective. Social science \& medicine (1982) 2018, 213:123-133. 
19. Kesavan K, Devaskar SU: Intrauterine Growth Restriction: Postnatal Monitoring and Outcomes. Pediatric clinics of North America 2019, 66(2):403-423.

20. Rodger MA, Carrier M, Le Gal G, Martinelli I, Perna A, Rey E, de Vries JI, Gris JC: Meta-analysis of low-molecular-weight heparin to prevent recurrent placenta-mediated pregnancy complications. Blood 2014, 123(6):822-828.

21. Roberge S, Nicolaides K, Demers S, Hyett J, Chaillet N, Bujold E: The role of aspirin dose on the prevention of preeclampsia and fetal growth restriction: systematic review and meta-analysis. American journal of obstetrics and gynecology 2017, 216(2):110-120.e116.

22. Inácio QAS, Araujo Júnior E: Perinatal Outcomes of Fetuses with Early Growth Restriction, Late Growth Restriction, Small for Gestational Age, and Adequate for Gestational Age. 2019, 41(12):688-696.

23. Vayssière C, Sentilhes L, Ego A, Bernard C, Cambourieu D, Flamant C, Gascoin G, Gaudineau A, Grangé $G$, Houfflin-Debarge $V$ et al: Fetal growth restriction and intra-uterine growth restriction: guidelines for clinical practice from the French College of Gynaecologists and Obstetricians. European journal of obstetrics, gynecology, and reproductive biology 2015, 193:10-18.

24. Lees C, Marlow N, Arabin B, Bilardo CM, Brezinka C, Derks JB, Duvekot J, Frusca T, Diemert A, Ferrazzi $\mathrm{E}$ et al: Perinatal morbidity and mortality in early-onset fetal growth restriction: cohort outcomes of the trial of randomized umbilical and fetal flow in Europe (TRUFFLE). Ultrasound in obstetrics \& gynecology : the official journal of the International Society of Ultrasound in Obstetrics and Gynecology 2013, 42(4):400-408.

25. Modi $\mathrm{N}$ : Time to bring fetal growth assessment into the 21st century. BJOG : an international journal of obstetrics and gynaecology 2016, 123(12):1989.

26. Lausman A, Kingdom J: Intrauterine growth restriction: screening, diagnosis, and management. Journal of obstetrics and gynaecology Canada : JOGC = Journal d'obstetrique et gynecologie du Canada : JOGC 2013, 35(8):741-748.

27. Bernardes TP, Broekhuijsen K, Koopmans CM, Boers KE, van Wyk L, Tajik P, van Pampus MG, Scherjon SA, Mol BW, Franssen MT et al: Caesarean section rates and adverse neonatal outcomes after induction of labour versus expectant management in women with an unripe cervix: a secondary analysis of the HYPITAT and DIGITAT trials. BJOG : an international journal of obstetrics and gynaecology 2016, 123(9):1501-1508.

28. Horowitz KM, Feldman D: Fetal growth restriction: risk factors for unplanned primary cesarean delivery. The journal of maternal-fetal \& neonatal medicine : the official journal of the European Association of Perinatal Medicine, the Federation of Asia and Oceania Perinatal Societies, the International Society of Perinatal Obstet 2015, 28(18):2131-2134. 


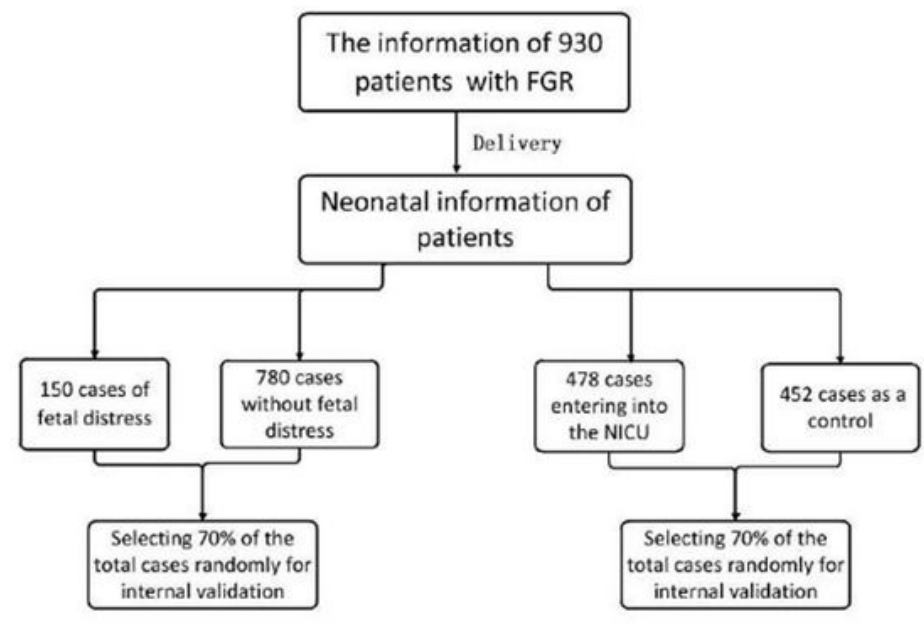

Figure 1A Study workflow of our research

Figure 1B

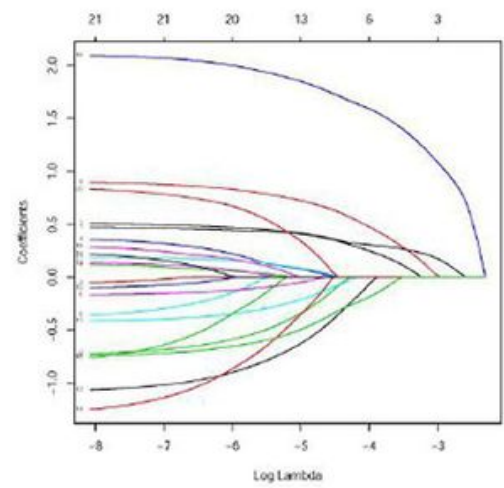

Figure 1D

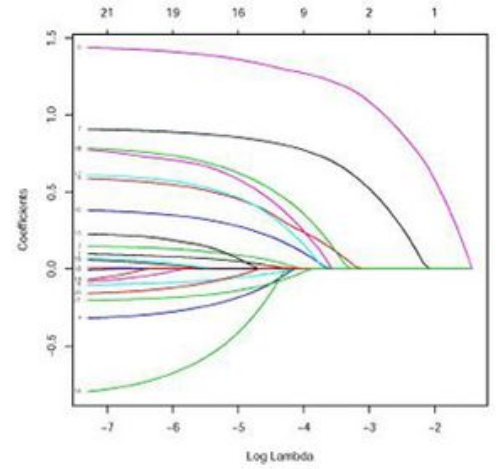

Figure $1 \mathrm{C}$

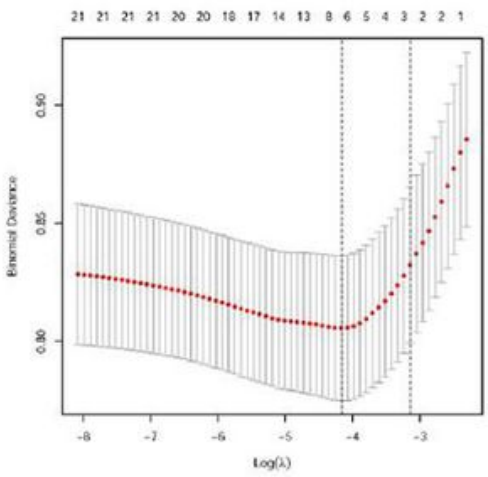

Figure 1E

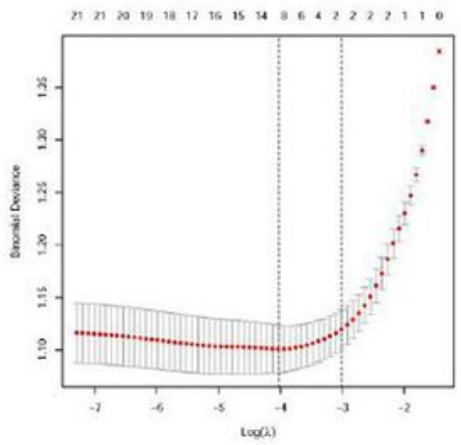

\section{Figure 1}

Study workflow and clinical characteristics selection using the LASSO regression analysis. LASSO coefficient profiles of the twenty-one features were plotted according to the log(lambda) sequence about fetal distress (Figure 1B) and admission to NICU (Figure 1D). The binomial deviance curves were 
produced versus log(lambda) about fetal distress (Figure 1C) and admission to NICU (Figure 1E). Dotted vertical lines were plotted at the optimal values by using the minimum criteria and the 1-standard error criteria.

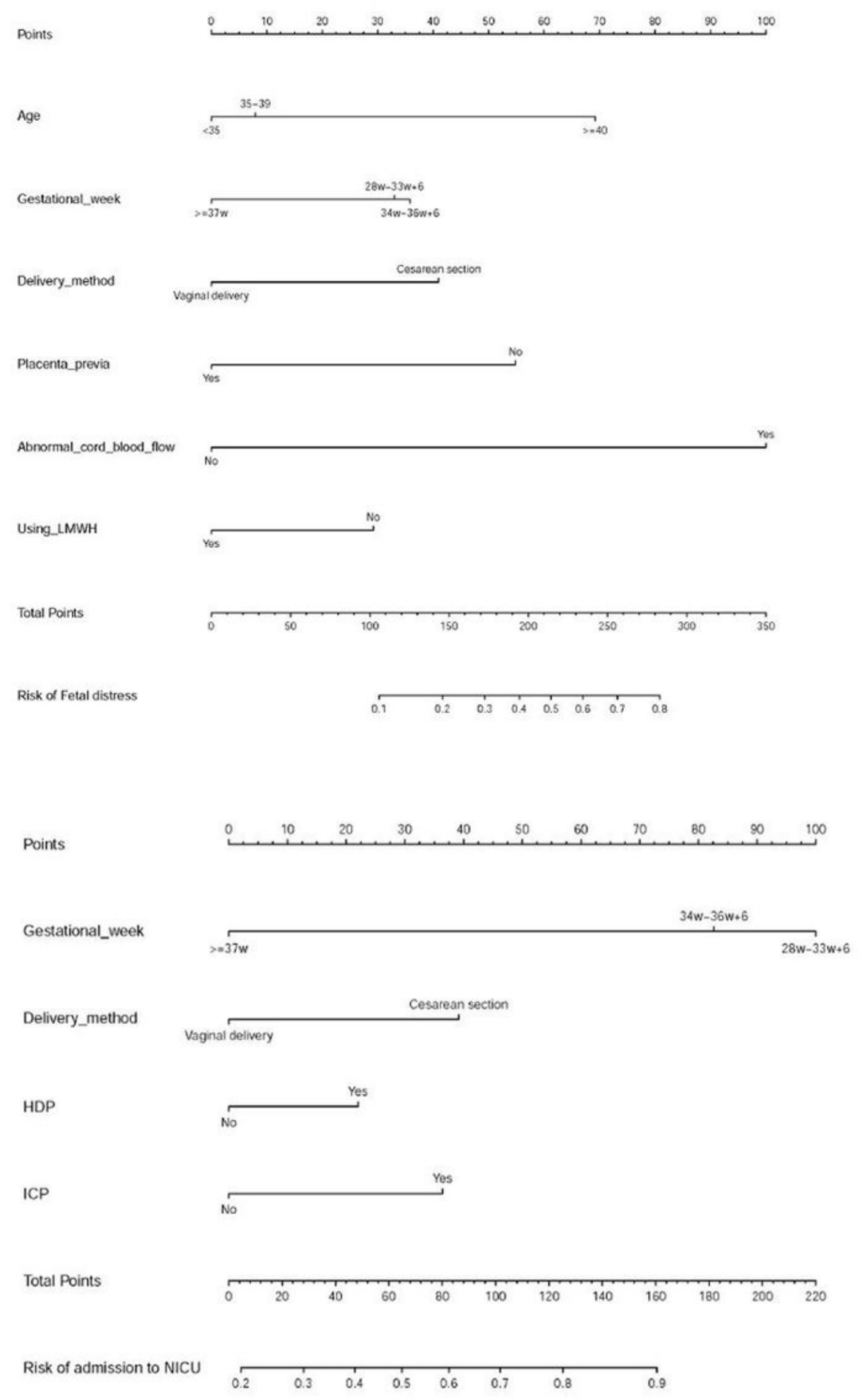

\section{Figure 2}


Figure $3 \mathrm{~A}$

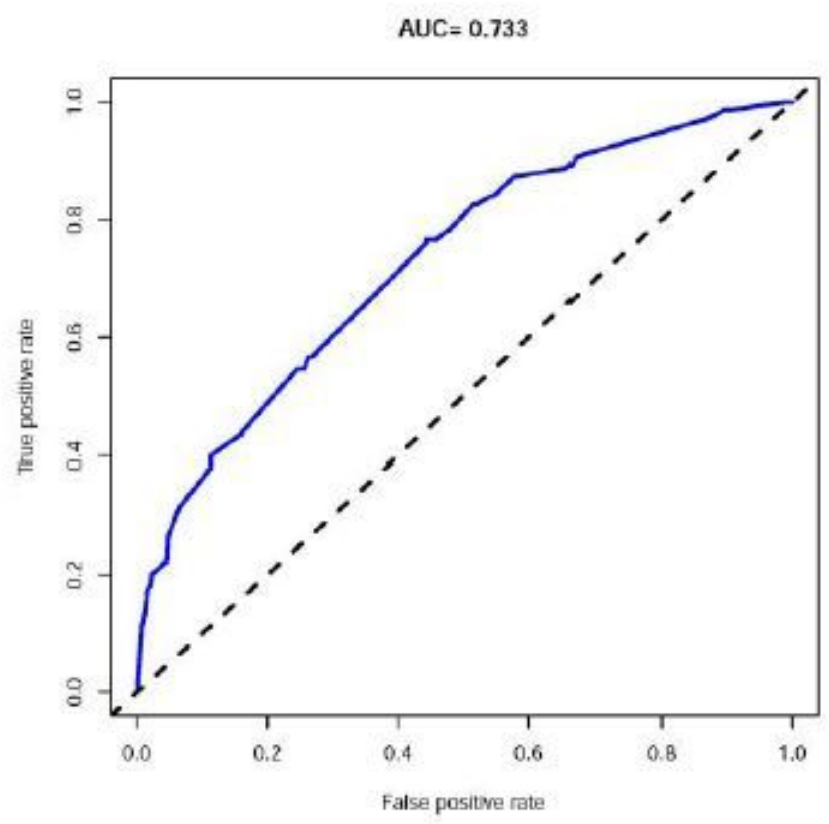

Figure $3 \mathrm{C}$

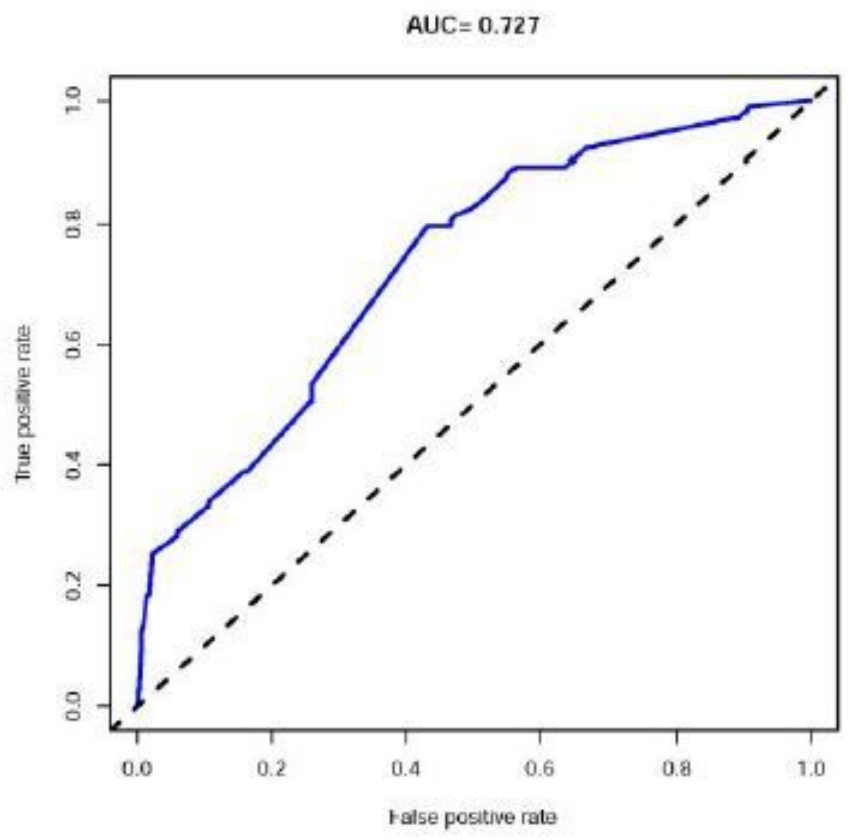

Figure 3B

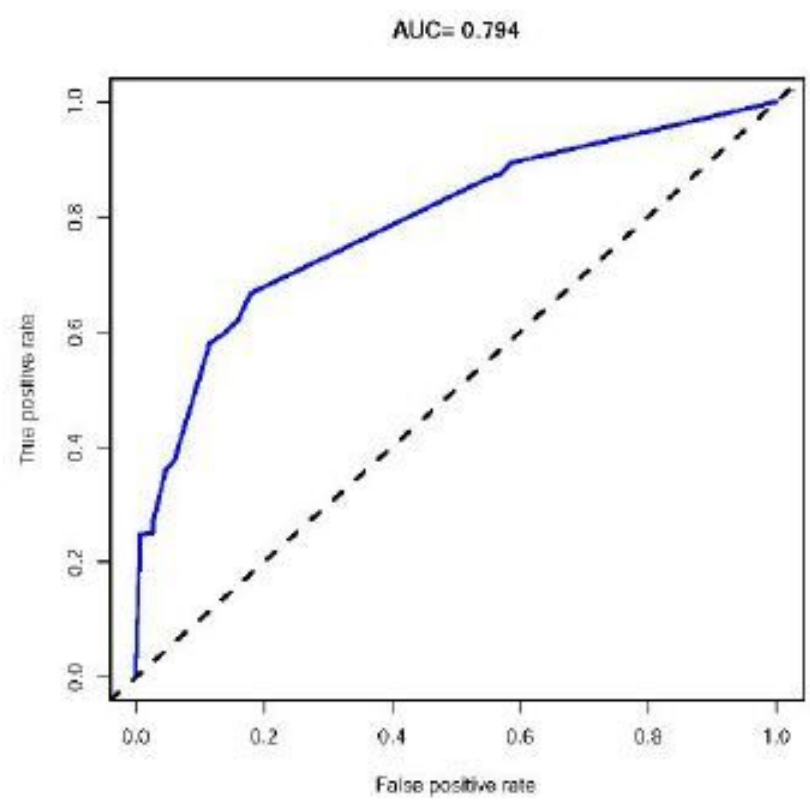

Figure 3D

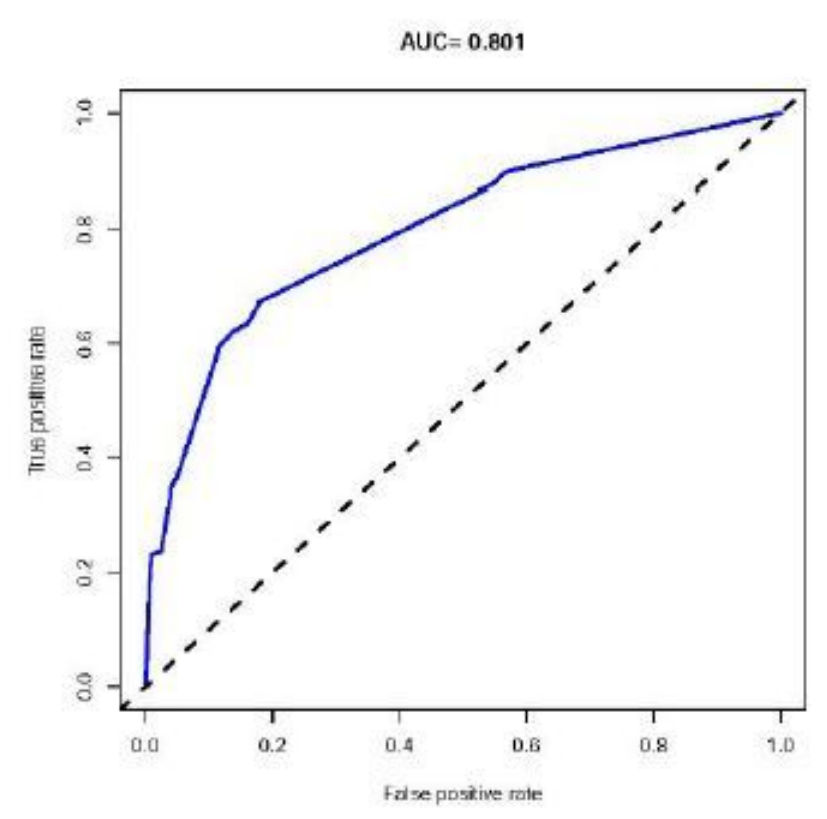

\section{Figure 3}

ROC curves for predicting the fetal distress risk and admission to NICU risk in the total and the internal Validation cases. (3A) ROC curve in the total cases of prediction model one. (3B) ROC curve in the total cases of prediction model two. (3C) ROC curve in the internal validation cases of prediction model one. (3D) ROC curve in the internal validation cases of prediction model two. 
Figure 4A

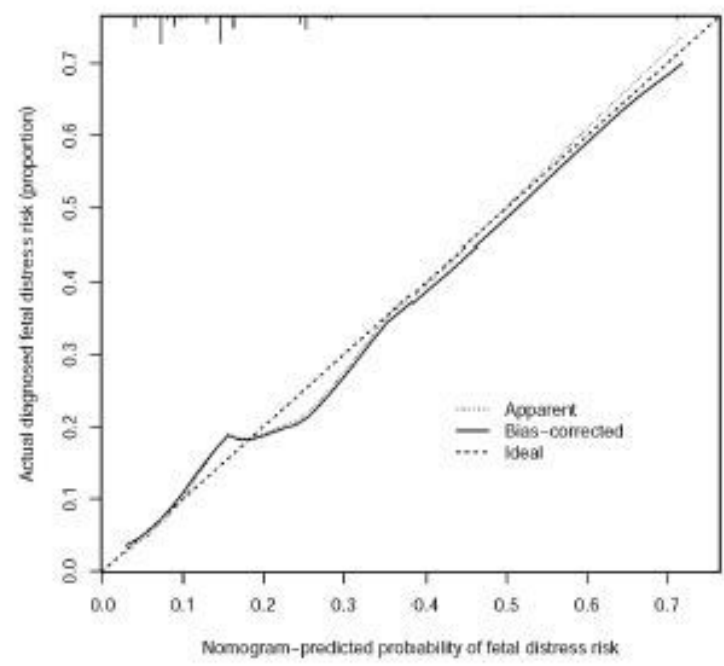

Figure 4C

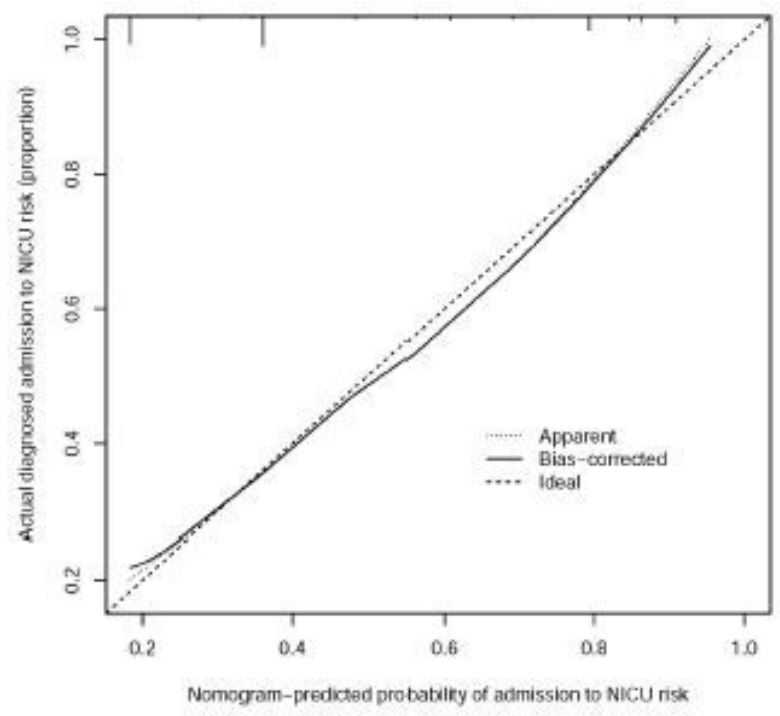

Figure 4B

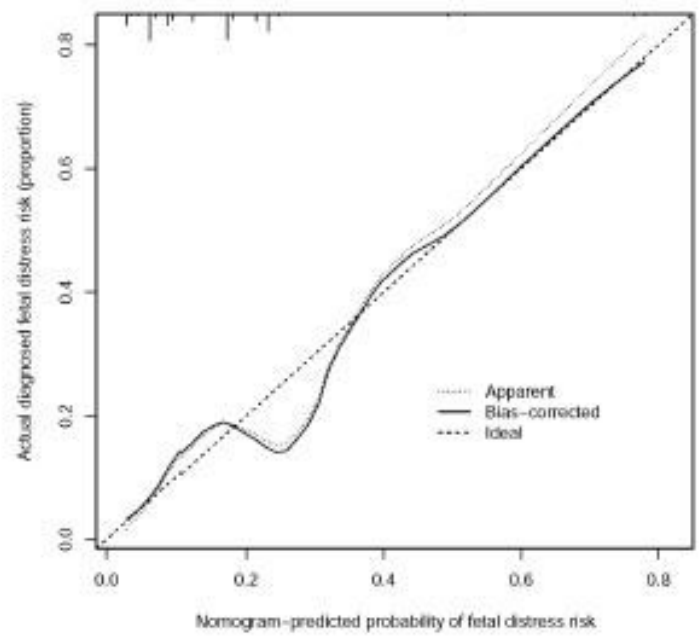

Figure 4D

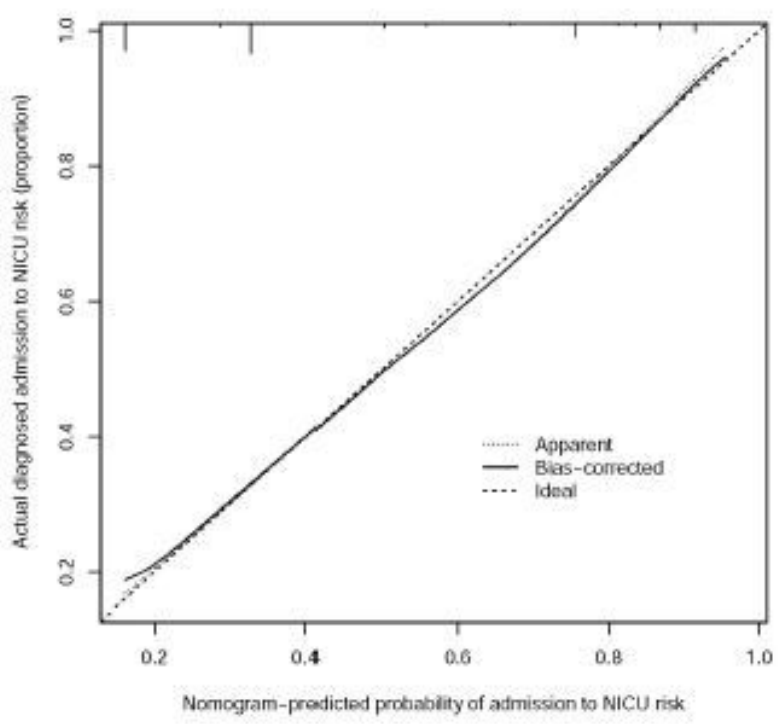

\section{Figure 4}

Calibration curves for predicting the fetal distress risk and admission to NICU risk in the total and the internal Validation cases. The x-axis measures the predicted fetal distress risk or admission to NICU risk. The $y$-axis represents the actual diagnosed fetal distress risk or admission to NICU risk. The diagonal dotted line represents an ideal prediction model. The solid line measures the performance of the nomogram, and a better prediction effect is that it is closer to the diagonal dotted line. 
Figure 5A

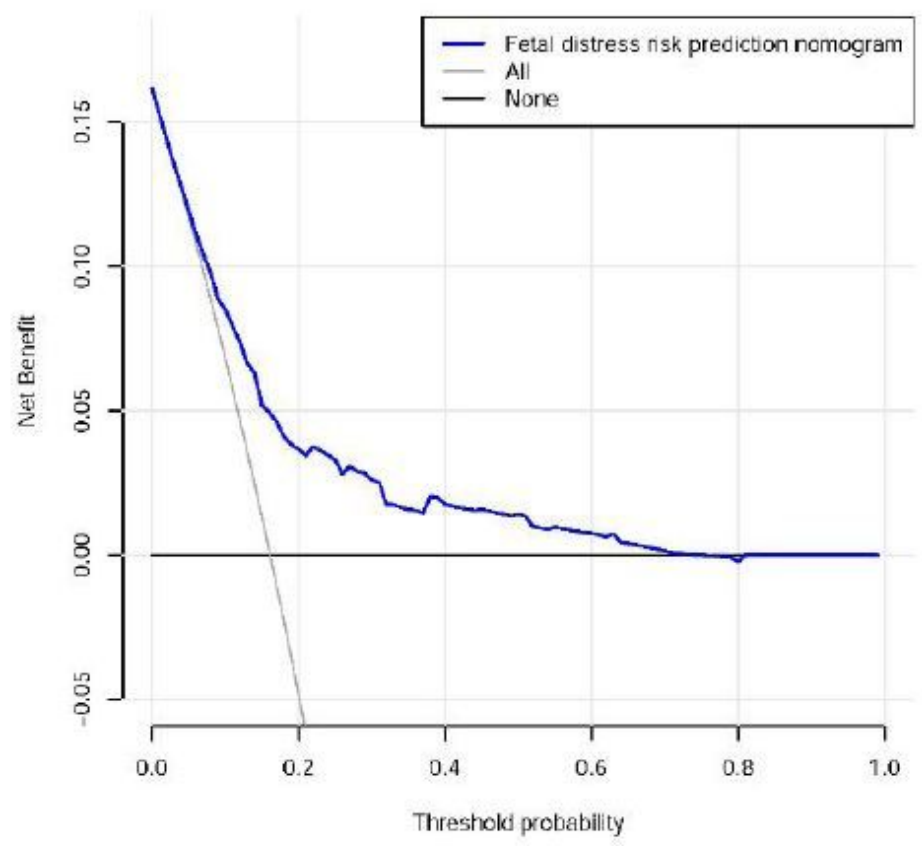

Figure 5B

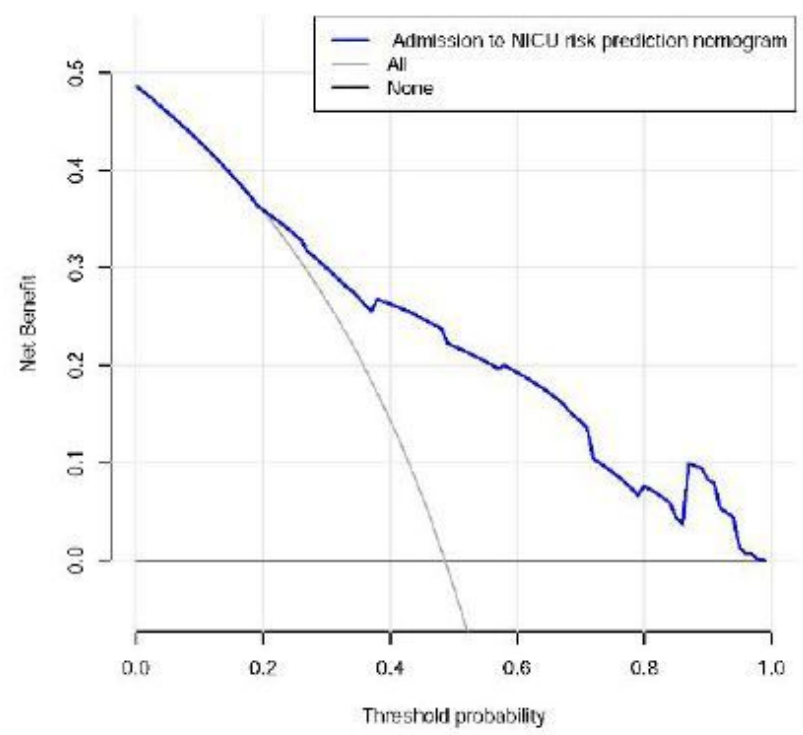

\section{Figure 5}

Decision curve analysis for the fetal distress risk nomogram(A) and admission to NICU risk nomogram(B) The $y$-axis represents the net benefit. The blue solid line measures the fetal distress risk nomogram(Figure 5A) and admission to NICU risk nomogram(Figure 5B). The thin solid line presumes all patients with FGR are the risk of fetal distress and admission to NICU. The thick solid line assumes that no patients with FGR are the risk of fetal distress and admission to NICU.

\section{Supplementary Files}

This is a list of supplementary files associated with this preprint. Click to download.

- Tables.pdf 